\title{
Temperature effect in the calibration of capacitive humidity sensors
}

\author{
Sergio A. Carvajal* and Ciro A. Sánchez \\ Instituto Nacional de Metrologia de Colombia, Bogota, Colombia
}

Received: 13 February 2018 / Accepted: 26 September 2018

\begin{abstract}
Capacitive hygrometers are widely used in industrial and environmental measurements. One of the limitations of these sensors is their temperature dependence. While in temperatures beyond $50{ }^{\circ} \mathrm{C}$ the effect has been proven to be significant, for standard conditions in calibration laboratories the magnitude of this dependence has not been rigorously studied. This paper presents the analysis and results of a study of the temperature influence in the calibration of capacitive hygrometers designed for monitoring environmental conditions. Ninety measurements were taken on 15 models of capacitive hygrometers using a two-pressure humidity generator between $30 \% \mathrm{rh}$ and $80 \% \mathrm{rh}$ and temperatures from 20 to $30^{\circ} \mathrm{C}$. A three-way analysis of variance (ANOVA) was used to evaluate the data. The results show that the calibration correction is independent of the temperature for the conditions considered; however, there is a correlation between the temperature and the devices, indicating that the gradients in temperature affect each technology of fabrication in a different way.
\end{abstract}

Keywords: temperature effect / capacitive hygrometers / ANOVA / relative humidity / calibration

\section{Introduction}

Humidity measurement is important in a wide variety of industrial processes, as a variable that affects the characteristics of a final product and as a variable that must be controlled. Currently, most of the hygrometers used (nearly 75\%) are capacitive [1]. The physical adsorption on surfaces is the phenomena that governs this measurement, so hysteresis, contaminants, concentration and temperature dependence should be evaluated [2]. The usual configuration is a hygroscopic material, generally a polymer, placed between the electrodes of a capacitor. When the polymeric film adsorbs water from the environment, the electrical permittivity changes and, consequently, the capacitance. By comparing it with the dry state of the material, the relative humidity can be determined [3].

The capacitive sensors respond to changes in relative humidity as well as to changes in temperature since the electrical properties depend sensitively on both. A curve fit is used to quantify the capacitance-humidity relationship based on a previously determined specific temperature dependence of the sensor [4]. The stability of the temperature dependence cannot be granted however, especially in industrial measurements. In most applica-

* Corresponding author: sacarvajal@inm.gov.co tions, the humidity measurements are not made in isothermal conditions so the temperature influence should be included in the uncertainty of the measurement. Unfortunately, the manufacturer does not usually provide information regarding the temperature effect or does not offer information on the entire interval of use. Heinonen [5] presents the temperature dependence between 10 and $60^{\circ} \mathrm{C}$ for a single impedance hygrometer, a difference from the reference value close to $5 \%$ was found. Fernicola et al. [6] evaluated the stability of 10 capacitive hygrometers at high temperatures concluding that at $80^{\circ} \mathrm{C}$, the errors greatly exceed the tolerances established by the manufacturer.

As a part of the mission of the National Metrology Institute of Colombia (INM), the Temperature and Humidity Laboratory disseminates measurement in the humidity field through the provision of calibration services. A service of special interest is the calibration of capacitive hygrometers, which are used in calibration laboratories to monitor the environmental conditions as a requirement of ISO/IEC 17025 [7]. In this application, conditions near to $25^{\circ} \mathrm{C}$ and $50 \% \mathrm{rh}$ are used. However, for this interval, there are no studies reporting the influence of the temperature dependence.

The objective of this paper is to evaluate the temperature effect upon humidity sensors based on measurements made in capacitive hygrometers designed to monitor environmental conditions for temperatures between 20 and $30^{\circ} \mathrm{C}$. The data are evaluated using a 
Table 1. Specification of the devices.

\begin{tabular}{|c|c|c|c|c|c|}
\hline Device & Accuracy $( \pm \%$ rh $)$ & $\begin{array}{l}\text { Measurement } \\
\text { interval (\%rh) }\end{array}$ & $\begin{array}{l}\text { Measurement } \\
\text { interval }\left({ }^{\circ} \mathrm{C}\right)\end{array}$ & $\begin{array}{l}\text { Time } \\
\text { responses }\end{array}$ & $\begin{array}{l}\text { Probe } \\
\text { location }\end{array}$ \\
\hline M1 & 2.5 & $0-100$ & -20 to 50 & 2 & Internal \\
\hline M2 & 4 (within 25 and $85 \%$ rh) & $10-99$ & -10 to 60 & - & External \\
\hline M3 & 1.5 (within 20 and $70 \%$ rh) & $0-100$ & $0-50$ & - & External \\
\hline M4 & 3.5 (within 20 and $80 \% \mathrm{rh}$ ) & $0-100$ & -20 to 50 & $<15$ & Internal \\
\hline M5 & 2.25 (within 20 and $80 \%$ rh) & $0-100$ & -35 to 80 & - & Internal \\
\hline M6 & 1 (within 0 and $90 \%$ rh) & $0-100$ & -40 to 60 & 8 & External \\
\hline M7 & 3 (within 10 and $70 \%$ rh) & $10-95$ & $0-60$ & - & External \\
\hline M8 & 1 (within 0 and $90 \%$ rh) & $0-100$ & -20 to 60 & 17 & External \\
\hline M9 & 2 & $0-95$ & -40 to 60 & - & External \\
\hline M10 & 2 & $0-100$ & -20 to 50 & - & Internal \\
\hline M11 & 1 (within 0 and $90 \%$ rh) & $0-100$ & -40 to 60 & 8 & Internal \\
\hline M12 & 1 (within 0 and $90 \%$ rh) & $0-100$ & -40 to 60 & 8 & External \\
\hline M13 & 3 (within 10 and $95 \% \mathrm{rh}$ ) & $0-95$ & -17 to 60 & - & External \\
\hline M14 & 3.5 (within 20 and $80 \%$ rh) & $0-100$ & -40 to 70 & - & Internal \\
\hline M15 & 1.8 & $0-100$ & $0-50$ & - & Internal \\
\hline
\end{tabular}

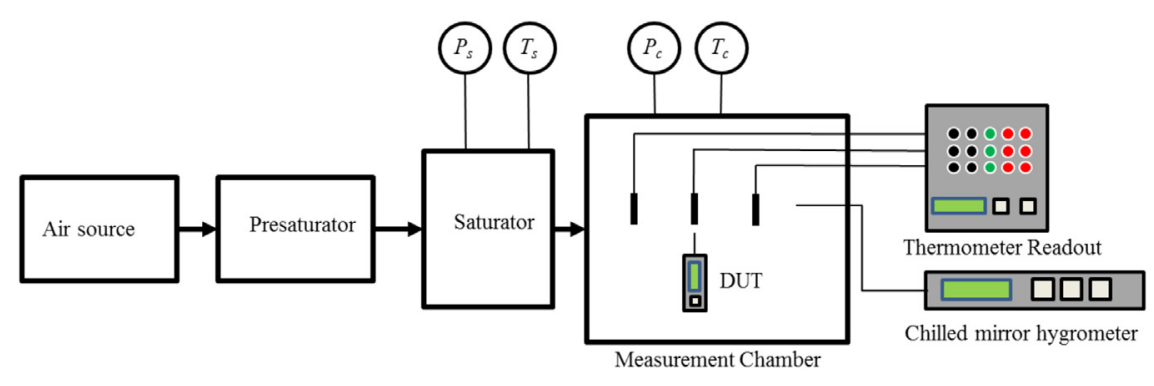

Fig. 1. Experimental bench.

three-way analysis of variance (ANOVA) and by comparing the temperature coefficient of each sensor. In order to avoid any commercially construed opinion, the brands and models of the evaluated items are codified (M1,M2, etc.).

\section{Measurements}

The study was performed using 15 capacitive sensors selected to represent the state of the art of environmental condition monitoring. The specifications are given in Table 1. The devices present both sensor probes separate from or attached to the signal processing system.

For each instrument, two calibrations were carried out at 20 and $30^{\circ} \mathrm{C}$. The calibrations were performed at increasing humidity for three levels (30, 50 and $80 \% \mathrm{rh}$ ). The lowest calibration value was repeated in order to ensure the stability of the devices throughout the test.

The reference measurement system is a two-pressure humidity generator that constitutes the humidity standard in the National Metrology Institute of Colombia (INM). The two-pressure method consists of the saturation of an air-water vapor mixture and its posterior isothermal expansion in a measurement chamber [8]. Under these conditions, the relative humidity can be calculated according to equation (1):

$$
\mathrm{HR}=\left(\frac{f_{\mathrm{w}}\left(T_{\mathrm{s}}, P_{\mathrm{s}}\right) \cdot e_{\mathrm{w}}\left(T_{\mathrm{s}}\right) \cdot P_{\mathrm{c}}}{f_{\mathrm{w}}\left(T_{\mathrm{c}}, P_{\mathrm{c}}\right) \cdot e_{\mathrm{w}}\left(T_{\mathrm{c}}\right) \cdot P_{\mathrm{s}}}\right) \times 100
$$

where $f_{\mathrm{w}}$ is the enhancement factor, $e_{\mathrm{w}}$ is the vapor pressure, $P_{\mathrm{c}}$ is the pressure in the chamber, $P_{\mathrm{s}}$ is the pressure in the saturator, $T_{\mathrm{s}}$ is the temperature in the saturator and $T_{\mathrm{c}}$ is the temperature in the chamber. Further details of the twopressure principle for humidity generation have been described in references $[8,9]$. The expanded uncertainty that can be achieved is about $0.8 \%$ rh [10]. Figure 1 shows the experimental bench of the measurement system and the device under test (DUT). A chilled mirror hygrometer was used to check the humidity, and three industrial platinum resistance thermometers were used to measure temperature and to monitor the uniformity and stability in the measurement chamber. 
Table 2. Three-way ANOVA calibration correction.

\begin{tabular}{lllll}
\hline & Sum of squares & Degrees of freedom & $F$ & p-value \\
\hline Humidity level & 89.64 & 2 & 246.31 & $<2 \times 10^{-16}$ \\
Device & 388.06 & 14 & 152.34 & $<2 \times 10^{-16}$ \\
Temperature & 0.01 & 1 & 0.05 & 0.84 \\
Humidity level:device & 71.05 & 28 & 13.95 & $3.13 \times 10^{-10}$ \\
Humidity level:temperature & 0.27 & 2 & 0.73 & 0.49 \\
Device:temperature & 15.94 & 14 & 6.26 & $2.03 \times 10^{-05}$ \\
Residuals & 5.09 & 28 & & \\
\hline
\end{tabular}

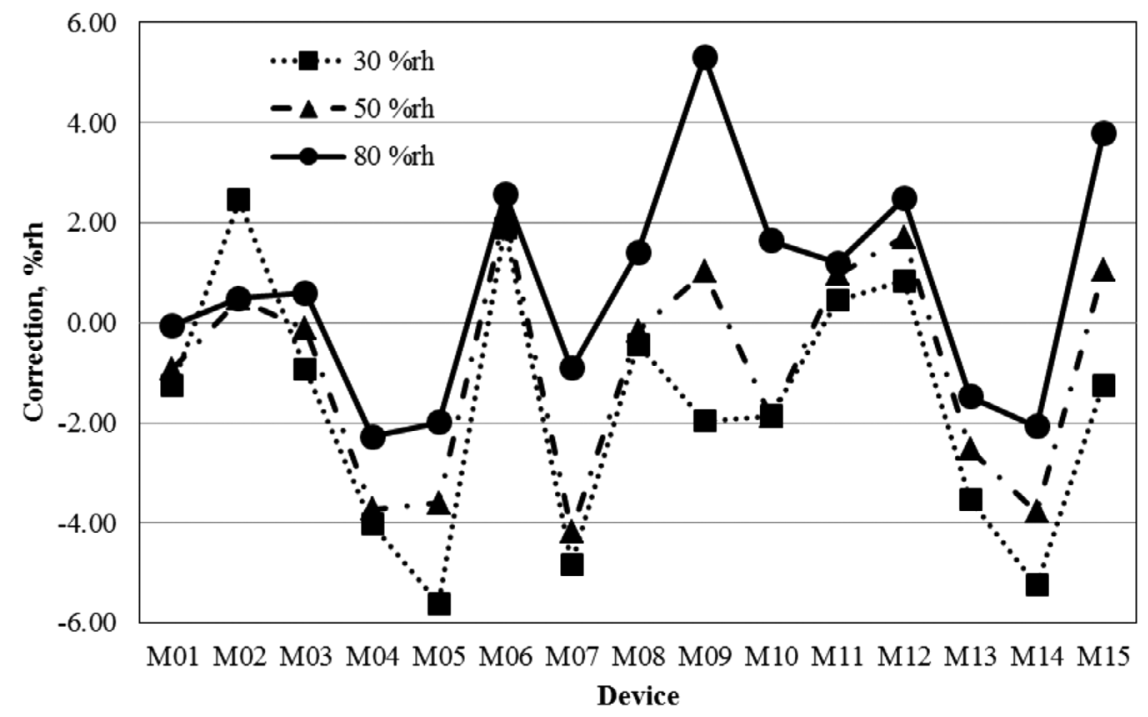

Fig. 2. Humidity: device effects.

In order to evaluate the temperature dependence and the interaction effects on the calibration, a statistical analysis was performed with a three-way ANOVA with a $95 \%$ confidence level. The factors considered were temperature $\left(20\right.$ and $\left.30^{\circ} \mathrm{C}\right)$, humidity level $(30,50$ and $80 \% \mathrm{rh}$ ) and the device (M1, M2, ..., M15). A total of 90 calibrations were carried out. In each measurement, the calibration correction was calculated according to equation (2):

$$
C_{\mathrm{HR}}=\mathrm{HR}_{m}-\mathrm{HR}_{x} .
$$

In this equation, $C_{\mathrm{HR}}$ is the calibration correction, $\mathrm{HR}_{m}$ is the humidity reference value and $\mathrm{HR}_{x}$ is the humidity of the device under test (DUT). Using the GUM framework [11], the uncertainty according to equation (2) can be expressed as follows:

$$
\begin{aligned}
u^{2}\left(C_{\mathrm{HR}}\right)= & u^{2}\left(\mathrm{HR}_{m}\right)+u^{2}\left(\mathrm{HR}_{x}\right)+u^{2}\left(\delta\left(\mathrm{HR}_{x}\right)_{\mathrm{Res}}\right) \\
& +u^{2}\left(\delta\left(\mathrm{HR}_{\mathrm{x}}\right)_{\mathrm{Hist}}\right)+C_{T} u^{2}\left(\delta\left(\mathrm{HR}_{m}\right)_{T}\right) .
\end{aligned}
$$

In this equation, $u^{2}\left(\mathrm{HR}_{m}\right)$ is the uncertainty of the humidity reference value, $u^{2}\left(\mathrm{HR}_{x}\right)$ is the uncertainty due to DUT repeatability, $u^{2}\left(\delta\left(\mathrm{HR}_{x}\right)_{\text {Res }}\right)$ is the uncertainty due to
DUT resolution, $u^{2}\left(\delta\left(\mathrm{HR}_{x}\right)_{\text {Hist }}\right)$ is the uncertainty due to DUT hysteresis, $u^{2}\left(\delta\left(\mathrm{HR}_{m}\right)_{T}\right)$ is the uncertainty due to the stability of the measurement chamber and $C_{T}$ is the temperature coefficient defined in equation (4).

$$
C_{T}=\frac{\Delta \mathrm{HR}}{\Delta T}
$$

\section{Results}

Table 2 provides the results of the three-way ANOVA. The effects plots are shown in Figures 2-4. Figure 2 shows that the device and the humidity level have a strong effect on the calibration correction, which is predictable based on the specification in Table 1. As can be seen in Figures 3 and 4 , there is not much difference between the plots of 20 and $30^{\circ} \mathrm{C}$, meaning that the temperature is not a significant factor. Also of note in Figure 3. is that the calibration correction increases with the humidity level. This behavior was in fact evidenced in all devices except in the least accurate one (M2). The $p$-value with $\alpha=0.05$ in Table 2 shows that humidity level:device and device:temperature 


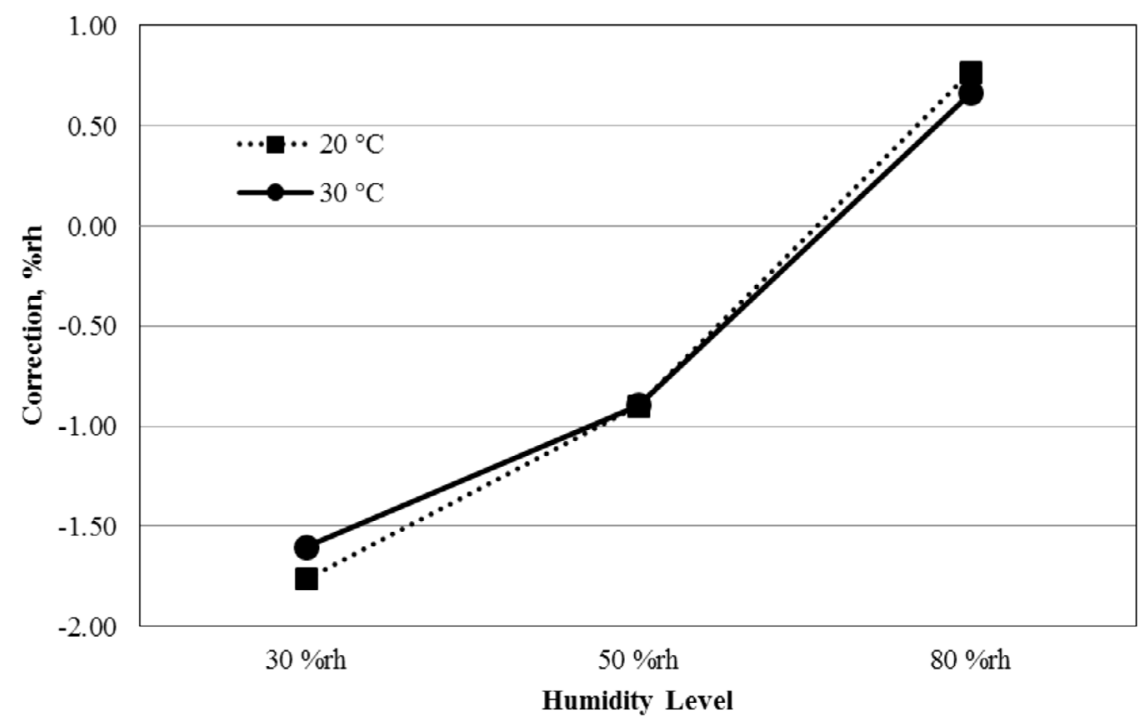

Fig. 3. Humidity: temperature effects.

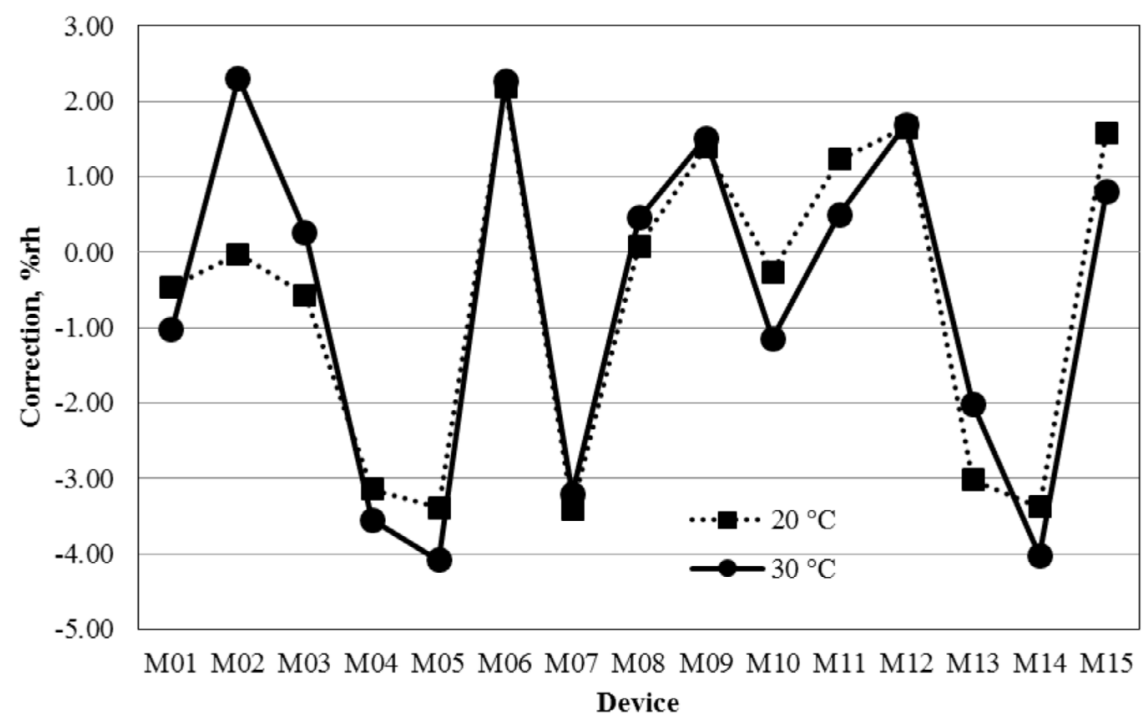

Fig. 4. Device: temperature effects.

interactions are significant, indicating that the design of the device affects temperature dependence. Finally, it was discovered that the humidity level:temperature interaction is not significant, which means that the influence of temperature is independent of the humidity level.

In order to quantify the differences in the temperature dependence, the temperature coefficient, defined by equation (4), was calculated for each device. Figures 5-8 show the coefficients for the 15 capacitive hygrometers. For the sake of readability, they were divided into four groups using the same scale. Differences were found in both the sign and magnitude of the coefficients, as well as for the dependence on the humidity level. Half of the models showed an increasing trend, four models showed linear behavior, and parabolic and exponential behaviors were

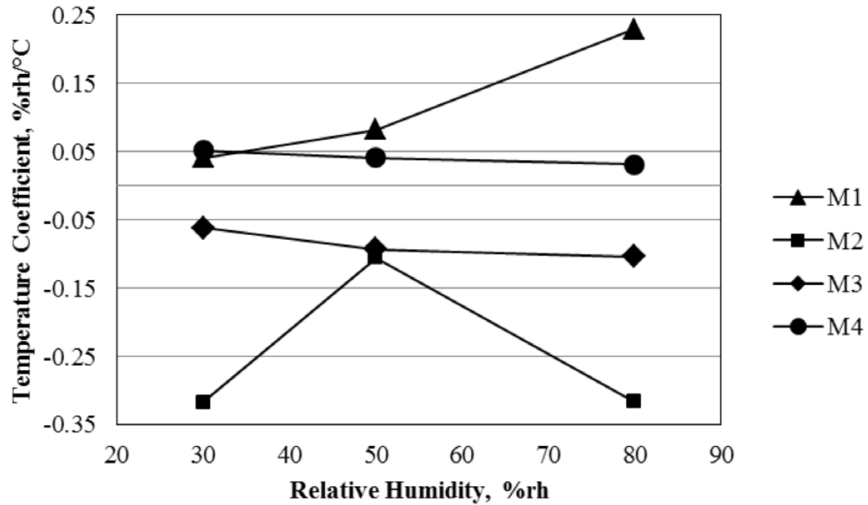

Fig. 5. Temperature coefficient (devices M1-M4). 


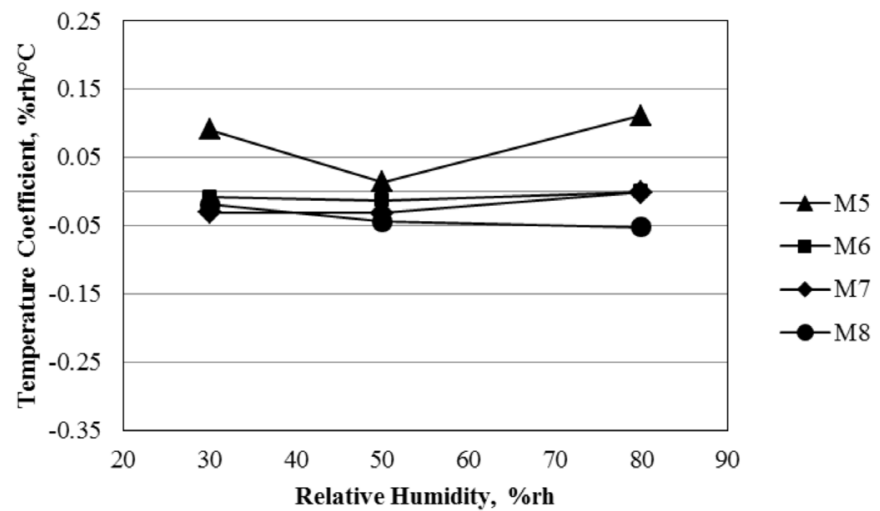

Fig. 6. Temperature coefficient (devices M5-M8).

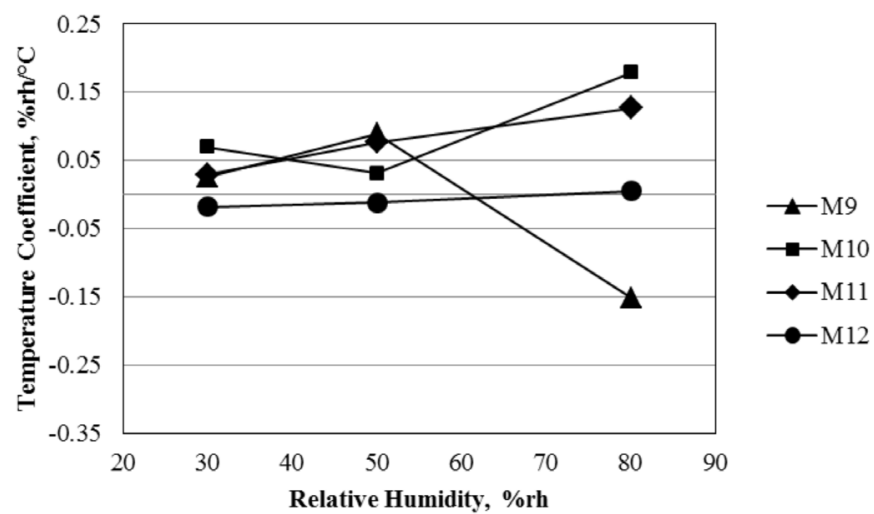

Fig. 7. Temperature coefficient (devices M9-M12).

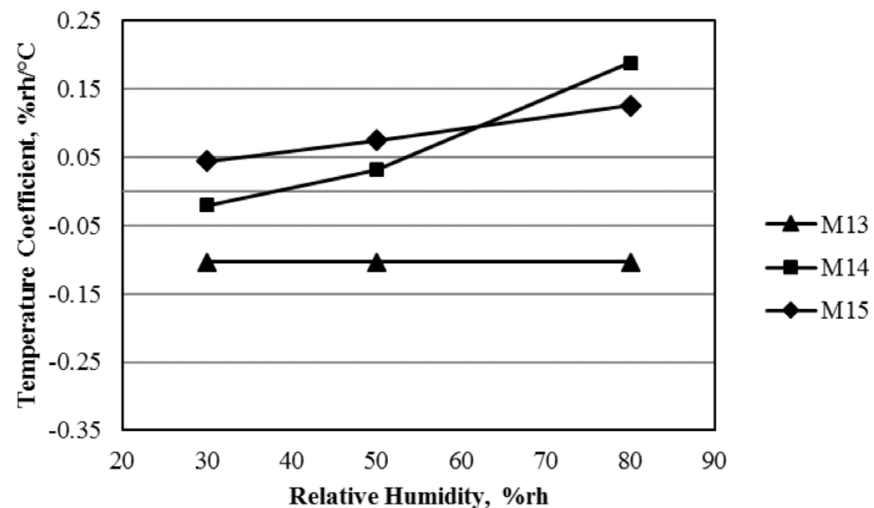

Fig. 8. Temperature coefficient (devices M13-M15). found in the others. The maximum and minimum absolute temperature coefficients were 0.32 and $0.01 \% \mathrm{rh} /{ }^{\circ} \mathrm{C}$, respectively.

A comparison between models M11 and M12 shows that the location of the probe impacts the temperature dependence. These devices are substantially the same except for the fact that in the first one, the probe is attached to the signal processing system, while in the second the sensing element can be separated from the readout. Figure 3 shows that the coefficient is lower in device M12 and negative at 30 and 50\% rh.

A two-way ANOVA was performed to evaluate the significance of humidity level and the device model in the temperature coefficient. The results are shown in Table 3. The main observations are the nonsignificant effect of the humidity level, consistent with the previous remark, and the strong dependency on the hygrometer model.

\section{Discussion}

One of the main applications of hygrometers is to monitor environmental conditions. In calibration laboratories, the temperature is usually kept between 20 and $30^{\circ} \mathrm{C}$. Under these conditions, it was found that the calibration correction is independent of temperature. Previous works show that for conditions as extreme as $150{ }^{\circ} \mathrm{C}$, the manufacturer specifications of capacitive sensors cannot be met [6]. However, for environmental conditions, the temperature effect can be neglected without compromising the accuracy of measurements.

Even though the temperature dependence is not significant in the calibration, it is useful to compare the temperature coefficient for the devices evaluated in order to judge the quality of the temperature compensating system implemented by the manufacturers. As is mentioned in reference [5], differences in both the sign and magnitude of the coefficients were found. Factors related to the design and construction of the sensor, such as vapor condensation, morphology in the porous material, and molecules dissociation affect the thermal response of the devices in different ways. One important observation is the independence of the humidity level with respect to the temperature coefficient. Although differences in the trends were found, the overall change in the temperature coefficient is negligible in comparison with the measurement uncertainties.

The influence of the probe location (internal/external) was evaluated using two hygrometers with equal specification. It was found that the temperature dependence is higher when the signal processing system is exposed to

Table 3. Two-way ANOVA temperature coefficient.

\begin{tabular}{llllll}
\hline & Sum of squares & Degrees of freedom & $F$ & $p$-value & $F$ critical value \\
\hline Device & 0.38 & 14 & 6.17 & $2.28 \times 10^{-5}$ & 2.06 \\
Humidity level & 0.01 & 2 & 0.92 & 0.41 & 3.34 \\
\hline
\end{tabular}


temperature changes; this is related to the fact that the associated electronics also present temperature sensitiveness.

\section{Conclusions}

The temperature effect in the calibration of 15 models of capacitive hygrometers was evaluated using the humidity standard in the National Metrology Institute of Colombia (INM) for temperatures between 20 and $30^{\circ} \mathrm{C}$ and for humidities between 30 and $80 \%$ rh.

The results indicate that the temperature effect is strongly dependent on manufacturer technology and independent of the humidity level. The effect in the calibration correction, however, is not significant. This means that approximating by excluding temperature dependence in the calibration of capacitive humidity sensors is valid for the input conditions studied.

\section{References}

1. Z.M. Rittersma, Recent achievements in miniaturised humidity sensors: a review of transduction techniques, Sens. Actuators A Phys. 96, 196-210 (2002)
2. N. Yamazoe, Y. Shimizu, Humidity sensors: principles and applications, Sens. Actuators 10, 379-398 (1986)

3. R. Wernecke, J. Wernecke, Industrial Moisture and Humidity Measurement: A Practical Guide (Wiley-VCH Verlag GmbH, Weinheim, Germany, 2013)

4. H. Smit, R. Kivi, H. Vömel, A. Paukkunen, Thin film capacitive sensors, in Monitoring Atmospheric Water Vapour, edited by N. Kämpfer (Springer, New York, NY, 2013), pp. 11-38

5. M. Heinonen, Uncertainty in humidity measurements, EUROMET Workshop P758, 2006

6. V. Fernicola, M. Banfo, L. Rosso, D. Smorgon, Investigation of capacitive-based relative humidity sensors and their stability at high temperature, Int. J. Thermophys. 29, 1668-1677 (2008)

7. ISO/IEC 17025:2005, General Requirements for the Competence of Testing and Calibration Laboratories, 2005

8. S. Hasegawa, J. W. Little, The NBS two-pressure humidity generator, mark 2, J. Res. NBS A 81A, 81-88 (1977)

9. J.G. Skabar, Uncertainty estimations for standard humidity generator of INTI, Int. J. Thermophys. 36, 2172-2184 (2015)

10. B. Hardy, Uncertainty analysis of the thunder scientific model2500 two-pressure humidity generator, 1998. Available at http://www.thunderscientific.com/tech info/index.html

11. BIPM IEC IFCC ILAC IUPAC OIML, Guide to the expression of uncertainty in measurement, JCGM 100:2008 (GUM), 2008. Available at http//www.bipm.org/

Cite this article as: Sergio A. Carvajal, Ciro A. Sánchez, Temperature effect in the calibration of capacitive humidity sensors, Int. J. Metrol. Qual. Eng. 9, 9 (2018) 